Check for updates

Received 8th October 2019

Accepted 15th November 2019

DOI: $10.1039 / c 9 r a 08142 a$

rsc.li/rsc-advances
Cite this: RSC Adv., 2019, 9, 39924

\section{Ultrasound-induced reactive oxygen species generation and mitochondria-specific damage by sonodynamic agent/metal ion-doped mesoporous silica†}

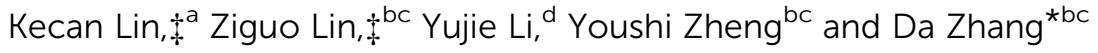

Designing tumor microenvironment (TME)-specific active nanoparticles with minimum side effects for synergistic cancer therapy has become a hot topic in the recent decades. Aiming at further enhancing the therapeutic efficacy, an in situ-induced mitochondrial dysfunction is a very promising strategy. To achieve these goals, a nano-sono-chemodynamic agent denoted as TPP-Cu@HMS, which integrated hematoporphyrin monomethyl ether (HMME), mPEG-NHS, triphenylphosphonium (TPP)-decorated mesoporous silica (MS) and coordinatively bound $\mathrm{Cu}^{2+}$ ions for mitochondria-specific sonodynamicchemodynamic therapy (SDT-CDT) of cancer, was designed. Upon the ultrasound (US) treatment, TPP$\mathrm{Cu}$ (aHMS can specifically target mitochondria and in situ generate ${ }^{1} \mathrm{O}_{2}$ against cancer cells. Specifically, to overcome the short lifespan of ${ }^{1} \mathrm{O}_{2}$, the released $\mathrm{Cu}^{2+}$ ions from TPP-CuaHMS could act as a Fentonlike agent to convert endogenous $\mathrm{H}_{2} \mathrm{O}_{2}$ to $\cdot \mathrm{OH}$ in the acidic environment of cancer cells, disrupt the mitochondrial membrane potential and lead to mitochondrial disintegration, which could systematically enhance the therapeutic efficiency of SDT. Therefore, we highlight the current strategy as a promising prospect for cancer therapy.

\section{Introduction}

Mitochondria as cellular power components play an important part in cell energy metabolism and survival in response to external stresses such as nutrient deprivation, ischemia and genotoxic agents. ${ }^{1-4}$ Damaged mitochondria can also induce a mitochondrial membrane potential change and subsequently cause mitochondria swelling/disintegration, cytochrome c and pro-apoptotic protein release, and eventually induce cell apoptosis/death. ${ }^{5,6}$ To control mitochondriaspecific damage, stimuli-responsive probes and smart nanocarrier systems have been designed recently, which are sensitive to endogenous ( $\mathrm{pH}$, redox chemistry and enzymes) and exogenous stimuli (NIR lasers and magnetic field). ${ }^{7-13}$ Among the various stimuli, ultrasound (mechanical waves

\footnotetext{
${ }^{a}$ The First Affiliated Hospital of Fujian Medical University, Fuzhou 350025, P. R. China ${ }^{b}$ The United Innovation of Mengchao Hepatobiliary Technology Key Laboratory of Fujian Province, Mengchao Hepatobiliary Hospital of Fujian Medical University, Fuzhou 350025, P. R. China. E-mail: zdluoman1987@163.com

'The Liver Center of Fujian Province, Fujian Medical University, Fuzhou 350025, P. R. China

${ }^{d}$ Reproductive Medicine Center, The Sixth Affiliated Hospital, Sun Yat-sen University, Guangzhou, 510000, P. R. China

$\dagger$ Electronic supplementary information (ESI) available. See DOI: 10.1039/c9ra08142a

‡ K. Lin and Z. Lin contributed equally to this work.
}

with a high penetrative power) as a new exogenous stimulus not only penetrates the depth of tumor tissues but also minimizes the side effects on the surrounding normal tissues..$^{14}$ Moreover, ultrasound sonodynamic therapy (SDT) as a promising non-invasive approach results in apoptosis in a variety of cancer cells including lymphocytes, myeloid leukemia and sarcoma cells through the activation of SDT agents to generate reactive oxygen species (ROS), cavitation, gas bubbles and hyperthermia. Especially in mitochondriaspecific damage, $\operatorname{ROS}\left({ }^{1} \mathrm{O}_{2}, \cdot \mathrm{OH}\right.$, etc. $)$ can lead to cell apoptosis/death efficiently. ${ }^{15}$ Nevertheless, the heterogeneity of TME can set several biological barriers for SDT, such as lower $\mathrm{pH}$ and hypoxia conditions, which are attributed to the disorganized vasculature inside tumors and the anaerobic respiration of tumor cells, ${ }^{16-18}$ increasing the risk of tumor relapse after SDT.

Recently, Fenton-like agent (such as $\mathrm{Cu}^{2+}, \mathrm{Mn}^{2+}$, and $\mathrm{Fe}^{2+}$ )based chemodynamic therapy (CDT) has been demonstrated for cancer therapy via the generation of $\cdot \mathrm{OH}$ from endogenous $\mathrm{H}_{2} \mathrm{O}_{2}$ in the most important physiological buffers to effectively induce DNA, protein and lipid damage, subsequently leading to cell apoptosis. ${ }^{18-21}$ Noteworthily, CDT is an oxygen-independent therapy that can overcome the hypoxia in TME and be easily combined with other therapeutic approaches such as PTT for enhanced cancer therapy according to our previous works. ${ }^{19,21}$ In this study, we introduced a new strategy combining SDT and 


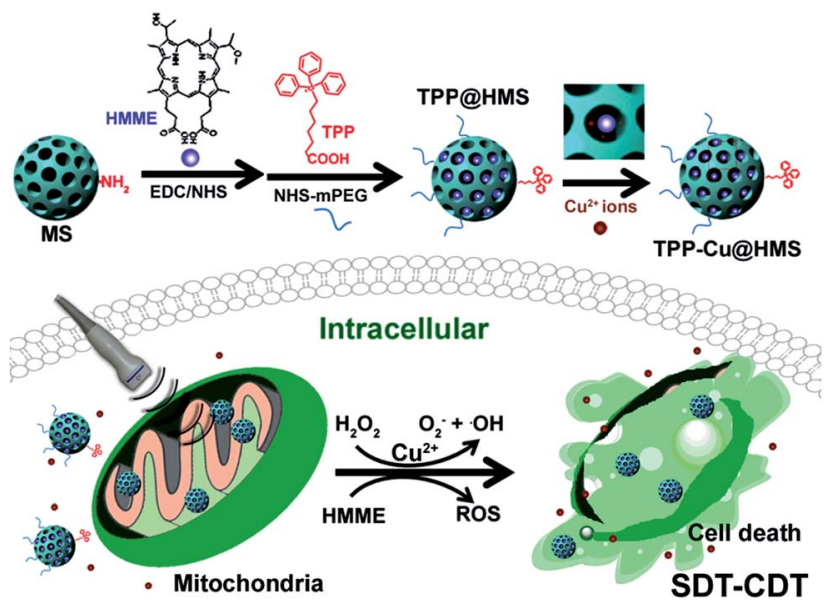

Scheme 1 Mitochondria-specific TPP-Cu@HMS with ROS burst for SDT-CDT synergy therapy. TPP-CuaHMS consists of TPP-COOH, $\mathrm{Cu}\left({ }^{\prime \prime}\right), \mathrm{HMME}$ and MS. TPP-Cu@HMS can specifically target mitochondria and in situ generate ${ }^{1} \mathrm{O}_{2}$ and $\cdot \mathrm{OH}$ upon exposure to ultrasound and $\mathrm{H}_{2} \mathrm{O}_{2}$ conditions for enhanced cancer therapy.

CDT for the mitochondria-specific therapy of cancer cells (Scheme 1). To achieve this goal, we used the FDA-approved SDT agent hematoporphyrin monomethyl ether (HMME) to covalently conjugate with triphenylphosphonium (TPP)-modified mesoporous silica as the SDT nanoagent (TPP@HMS) and subsequently coordinated it with $\mathrm{Cu}^{2+}$ (denoted as TPP$\mathrm{Cu}$ @HMS) for the in situ generation of ${ }^{1} \mathrm{O}_{2}$ and $\cdot \mathrm{OH}$ in mitochondria upon $\mathrm{H}_{2} \mathrm{O}_{2} /$ US for SDT-CDT.

\section{Experimental}

Materials

Mesoporous silica (MS) with an average diameter of $71 \pm 2.1 \mathrm{~nm}$ and pore diameter of $13.3 \mathrm{~nm}$, triphenylphosphonium (TPP$\mathrm{COOH}$ ) and succinimidyl propionic acid PEG (NHS-mPEG) were purchased from Xi'an Ruixi Company. N-Hydroxysuccinimide (NHS), hematoporphyrin monomethyl ether (HMME), $\quad N$-(3-dimethylamino-propyl)- $N$-ethylcarbodiimide hydrochloride (EDC) and $\mathrm{CuCl}_{2}$ were obtained from J\&K Scientific. The Cell Counting Kit (CCK-8) and LIVE/DEAD Viability/ Cytotoxicity Kit were obtained from Dojindo China Co., Ltd. 3Aminopropyltrimethoxysilane (APTES) and 2,7-dichlorodihydrofluoresceindiacetate (DCFH-DA) were purchased from Sigma-Aldrich. Deionized water with a resistivity of $18.2 \mathrm{M} \Omega \mathrm{cm}$ was obtained from a Milli-Q Gradient System (Millipore, Bedford, MA, U.S.).

\section{Cell culture}

The liver cancer cells line (HepG2 cells) was obtained from ATCC (Manassas, VA). The cells were cultured in RPMI 1640 medium (contained 10\% FBS) and penicillin-streptomycin (100 IU $\mathrm{mL}^{-1}$, Cellgro, Manassas, VA) at $37{ }^{\circ} \mathrm{C}$ in a humidified incubator containing $5 \% \mathrm{CO}_{2}$.

\section{Synthesis of the TPP-Cu@HMS}

Initially, $10 \mathrm{mg}$ of MS in ethanol was mixed with APTES $(50 \mu \mathrm{L})$ under reflux for $24 \mathrm{~h}$. Afterwards, $\mathrm{MS}-\mathrm{NH}_{2}$ was centrifuged and $1 \mathrm{mg}$ of HMME and $2 \mathrm{mg}$ of TPP-COOH were added to the above mixture solution containing EDC/NHS $\left(10 \mathrm{mg} \mathrm{mL}^{-1}\right)$ for $24 \mathrm{~h}$. Then, the resulting products TPP@HMS was centrifuged and washed 3 times with ethanol. Afterwards, TPP@HMS was mixed with NHS-mPEG for $24 \mathrm{~h}$, and purified by centrifugation, and washed 3 times with DI water. The dose of HMME in TPP@HMS was calculated by subtracting the absorbance at $400 \mathrm{~nm}$ of HMME from its standard curve for concentrations ranging from $1-14 \mu \mathrm{g} \mathrm{mL}{ }^{-1}\left(y=0.141 x+0.118, R^{2}=0.996\right)$. After that, the TPP@HMS solution was mixed with $\mathrm{CuCl}_{2}\left(\mathrm{pH} 7.4,10 \mathrm{mg} \mathrm{mL} \mathrm{m}^{-1}\right)$ and stirred for $12 \mathrm{~h}$. The final product of TPP-Cu@HMS was obtained by centrifugation, and it was stored at $4{ }^{\circ} \mathrm{C}$ for further use.

\section{Characterization of TPP-Cu@HMS}

Scanning transmission electron microscopy (STEM) with highangle annular dark-field and transmission electron microscopy (TEM) were conducted on a JEM-2010 electron microscope (JEOL, Japan). UV-vis spectra of TPP@HMS were investigated using a Spectro Max M5e (Germany). Fluorescence spectra were measured using an Agilent Cary Eclipse fluorescence spectrophotometer (Santa Clara, USA).

\section{The intracellular location of TPP@HMS}

To evaluate the subcellular location of TPP@HMS, HepG2 cells were first seeded in $35 \mathrm{~mm}$ glass bottom Petri dishes with $5 \times 10^{4}$ cells at $37{ }^{\circ} \mathrm{C}$ in a $5 \% \mathrm{CO}_{2}$ atmosphere for $24 \mathrm{~h}$ incubation. Then, HepG2 cells containing TPP@HMS were coincubated for $6 \mathrm{~h}$, and washed with PBS solution ( $\mathrm{pH} 7.4$ ) to remove the free TPP@HMS. Afterwards, the treated cells were co-stained with MitoTracker Red (500 nM) for $15 \mathrm{~min}$ and washed with PBS buffer solution. Subsequently, the cells were imaged via CLSM at $405 \mathrm{~nm}$ for HMME (TPP@HMS) and $561 \mathrm{~nm}$ for MitoTracker Red. Moreover, to investigate the morphology and mitochondrial membrane potential, the cells were then treated with or without US $\left(0.75 \mathrm{~W} \mathrm{~cm}^{-2}, 1 \mathrm{WHz}\right)$ for 3 min and then treated with the JC-1 dye detector kit according to the commercial protocol, and real-time imaged by CLSM.

\section{The intracellular ROS generation and cytotoxicity of TPP- Cu@HMS}

The ROS generation by TPP-Cu@HMS with or without US treatment was detected using DCFH-DA as the ROS indicator. Firstly, HepG2 cells were seeded in a 96-well plate with a density of $1 \times 10^{5}$ per well and co-incubated a humid atmosphere containing $5 \% \mathrm{CO}_{2}$ for $24 \mathrm{~h}$. Then, the above medium was refreshed with a new culture including TPP$\mathrm{Cu} @ H M S$ and co-incubated for $6 \mathrm{~h}$. Next, the cancer cells were treated with ROS detector $(100 \mu \mathrm{M}$, DCFH-DA) for $30 \mathrm{~min}$. Subsequently, the cells were washed with PBS buffer and then treated with or without US $\left(0.75 \mathrm{~W} \mathrm{~cm}^{-2}, 1 \mathrm{WHz}\right)$ for $3 \mathrm{~min}$, 
and imaged by CLSM. To evaluate the SDT killing effects of TPP-Cu@HMS, HepG2 cells were seeded into $35 \mathrm{~mm}$ glass bottom Petri dishes at a density of $5 \times 10^{4}$ cells at $37^{\circ} \mathrm{C}$ in a $5 \%$ $\mathrm{CO}_{2}$ atmosphere for $24 \mathrm{~h}$. Then, the HepG2 cells were coincubated with TPP@HMS for $6 \mathrm{~h}$, then washed with PBS buffer ( $\mathrm{pH} 7.4$ ) to remove the free TPP@HMS, and finally treated with $1 \mathrm{WHz}$ US $\left(0.75 \mathrm{~W} \mathrm{~cm}^{-2}, 1 \mathrm{WHz}\right)$ for $3 \mathrm{~min}$. The treated cells were stained by propidium iodide (PI) and imaged using a fluorescence microscope. Next, to quantitative measure the cytotoxicity of TPP@HMS upon US treatment, the CCK8 assay was performed according to our previously published protocols. ${ }^{18-21}$

\section{Statistical analysis}

Statistical analysis of CCK8 data was investigated through the two-tailed paired Student's $t$-test or one-way of variance
(ANOVA) protocol, where $* p<0.05,{ }^{*} p<0.01,{ }^{* * *} p<0.001$. The data is presented as the mean $\pm \mathrm{SD}$ from at least three experiments.

\section{Results and discussion}

Hemoporfin ${ }^{\circledR}$ is a commonly used PDT agent due to its satisfactory optical properties and ROS quantum yield (approximately 0.6). ${ }^{21}$ However, the metabolism of hydrophilic Hemoporfin ${ }^{\circledR}$ in clinic is very fast in the body, and thus it always suffers from a short retention time in tumors. ${ }^{21,22}$ In this study, we chose the hydrophobic HMME to covalently conjugate with MS as a new SDT nanoagent and decorated mPEG for enhancement in its circulation half-time. Firstly, the obtained MS with an average diameter of $71 \pm 2.1 \mathrm{~nm}$ and pore diameter of $13.3 \mathrm{~nm}$ was modified with 3-aminopropyltrimethoxysilane (APTES) to gain the amino $\left(-\mathrm{NH}_{2}\right)$

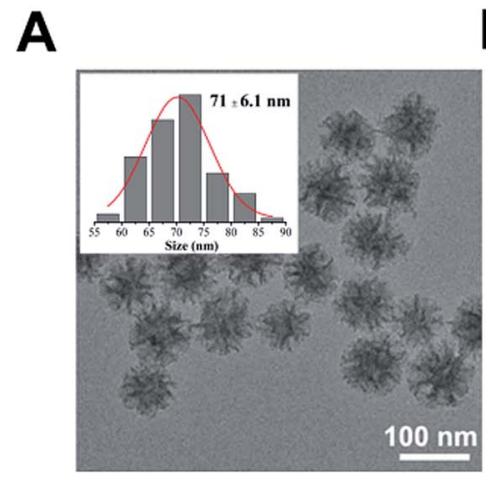

D

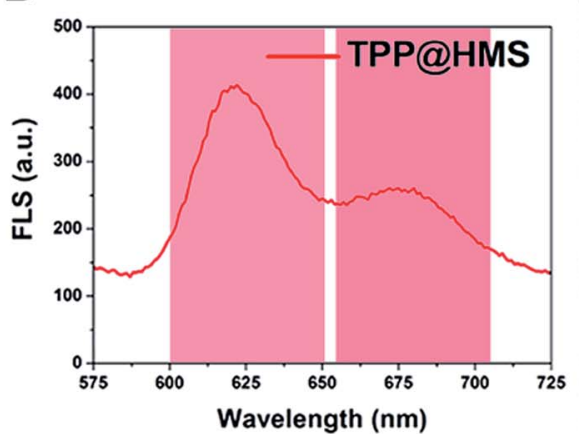

B

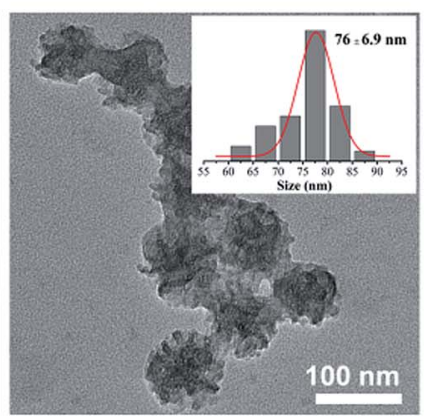

E

C
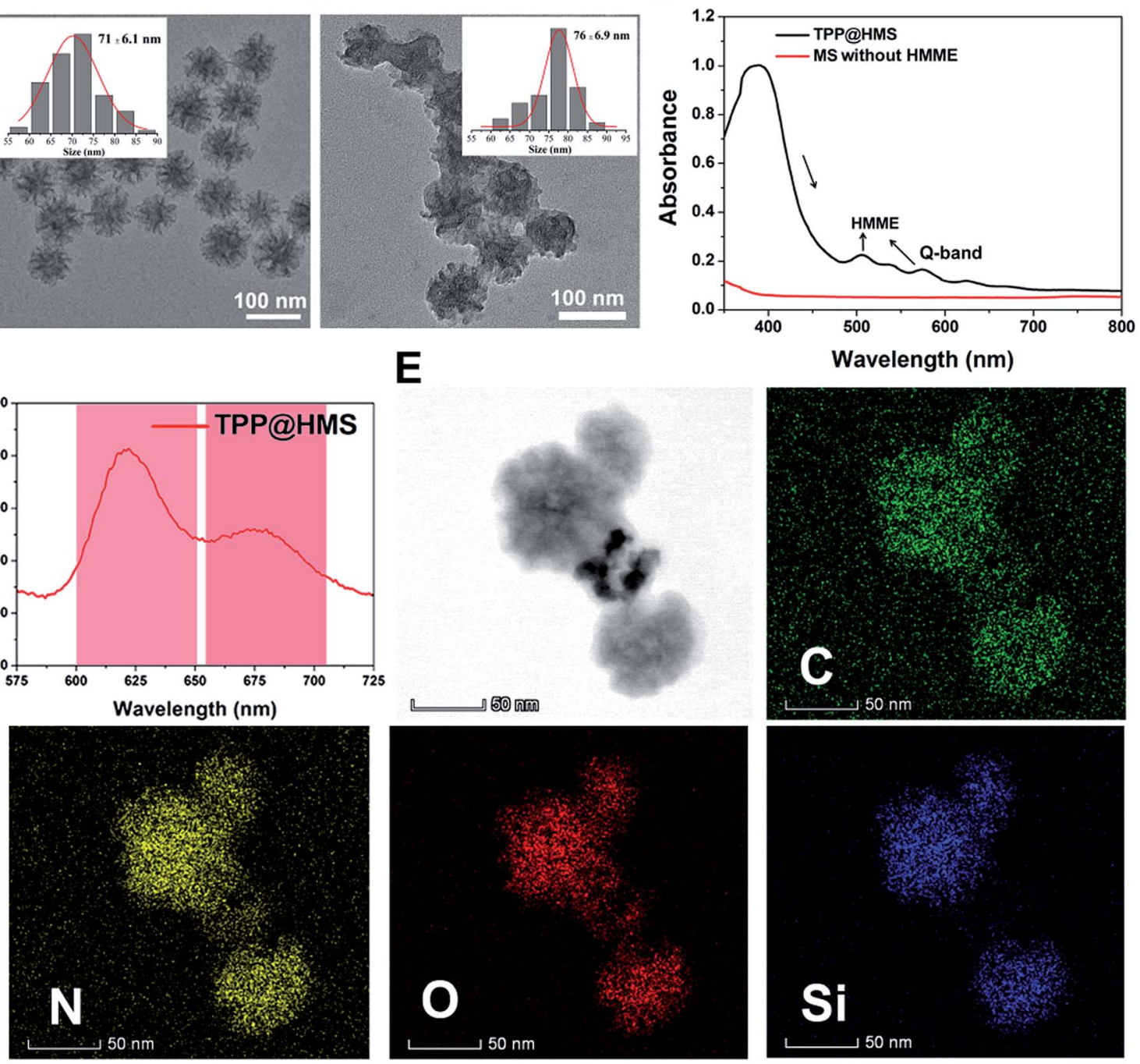

Fig. 1 (A) TEM image of MS-NH2 and (B) TPP@HMS, and the inset image is the size distribution of MS-NH2 and TPP@HMS, respectively. (C) UVvis-NIR spectra of TPPQHMS and MS-NH2. (D) Fluorescence spectrum of TPPQHMS (ex $391 \mathrm{~nm}$ ). (E) STEM elemental mapping of C, N, O and Si edge signals of TPP@HMS. 
group on the surface of MS. The morphology of MS- $\mathrm{NH}_{2}$ was evaluated via transmission electron microscopy (TEM). As shown in Fig. 1A and the inset picture, $\mathrm{MS}-\mathrm{NH}_{2}$ showed good monodispersity and a spherical shape, with an average diameter of $71 \pm 6.1 \mathrm{~nm}$. After modification with APTES, HMME was then covalently conjugated to $\mathrm{MS}-\mathrm{NH}_{2}$ via the amido bond to form a new SDT agent (named HMS). Afterwards, the mitochondria-specific ligand of TPP-COOH and NHS-mPEG were conjugated with the surface of HMS through amido bonds (refer to TPP@HMS). The TEM image of TPP@HMS showed the typical spherical shape and the average diameter of $76 \pm 6.9 \mathrm{~nm}$ (Fig. 1B). The UV-vis-NIR spectrum obtained on a Spectro-Max M5e was further investigated to confirm the components of the final product. As shown in Fig. 1C, four distinct peaks in the wavelength range of 450-750 nm at $506 \mathrm{~nm}, 544 \mathrm{~nm}, 576 \mathrm{~nm}$ and $622 \mathrm{~nm}$ (Qband) were observed clearly compared to $\mathrm{MS}-\mathrm{NH}_{2}$ without HMME conjugation, and the results are consistent with the typical absorption peaks of HMME. ${ }^{22}$ The emission spectrum of TPP@HMS demonstrates that its fluorescence emission is long in the NIR range (600 to $700 \mathrm{~nm}$ ) (excited by $391 \mathrm{~nm}$ ). To further confirm its components, STEM with high-angle annular dark-field was performed. The final data suggests the presence of $\mathrm{N}, \mathrm{O}, \mathrm{C}$ and $\mathrm{Si}$ homogenously distributed throughout TPP@HMS (Fig. 1E).
Mitochondria-specific damage of cancer cells is an effective strategy with remarkable therapeutic potency in cancer therapy. ${ }^{\mathbf{1 - 4}}$ To investigate the mitochondrial targeting ability of HMS with decoration of TPP-COOH, HepG2 cells as a typical cancer cell model were chosen. As shown in Fig. 2A and B, the confocal laser scanning microscopy (CLSM) images showed good overlap between TPP@HMS with red fluorescence and MitoTracker Red with green fluorescence (pseudo-color) after co-incubation for $6 \mathrm{~h}$, and Pearson's correlation coefficient (PCCs) was calculated to be 0.86 using the ImageJ software. PBS-treated HepG2 cells stained with MitoTracker Red were as a control. To achieve excellent therapeutic efficiency for the treatment of cancer, in situ mitochondria ROS generation is a very promising approach. ${ }^{5,6}$ Inspired by this, we checked the ${ }^{1} \mathrm{O}_{2}$ generation ability of our prepared TPP@HMS. The ${ }^{1} \mathrm{O}_{2}$ indicator 1,3-diphenylisobenzofuran (DPBF) was used to measure ${ }^{1} \mathrm{O}_{2}$ upon $1 \mathrm{WHz}$ US $\left(1 \mathrm{~W} \mathrm{~cm}{ }^{-2}\right) .{ }^{24}$ The change in optical density was investigated by monitoring the US-vis spectrum. As shown in Fig. 3A and B, the absorbance of the DPBF solution containing TPP@HMS ranging from 350 to $500 \mathrm{~nm}$ significantly decreased after US, and the decrease was time dependent, suggesting the excellent ${ }^{1} \mathrm{O}_{2}$ generation ability of TPP@HMS. In contrast, the DPBF solution without TPP@HMS did not obviously change under the same US power condition (Fig. 3B and C). Moreover, the mitochondrial

A
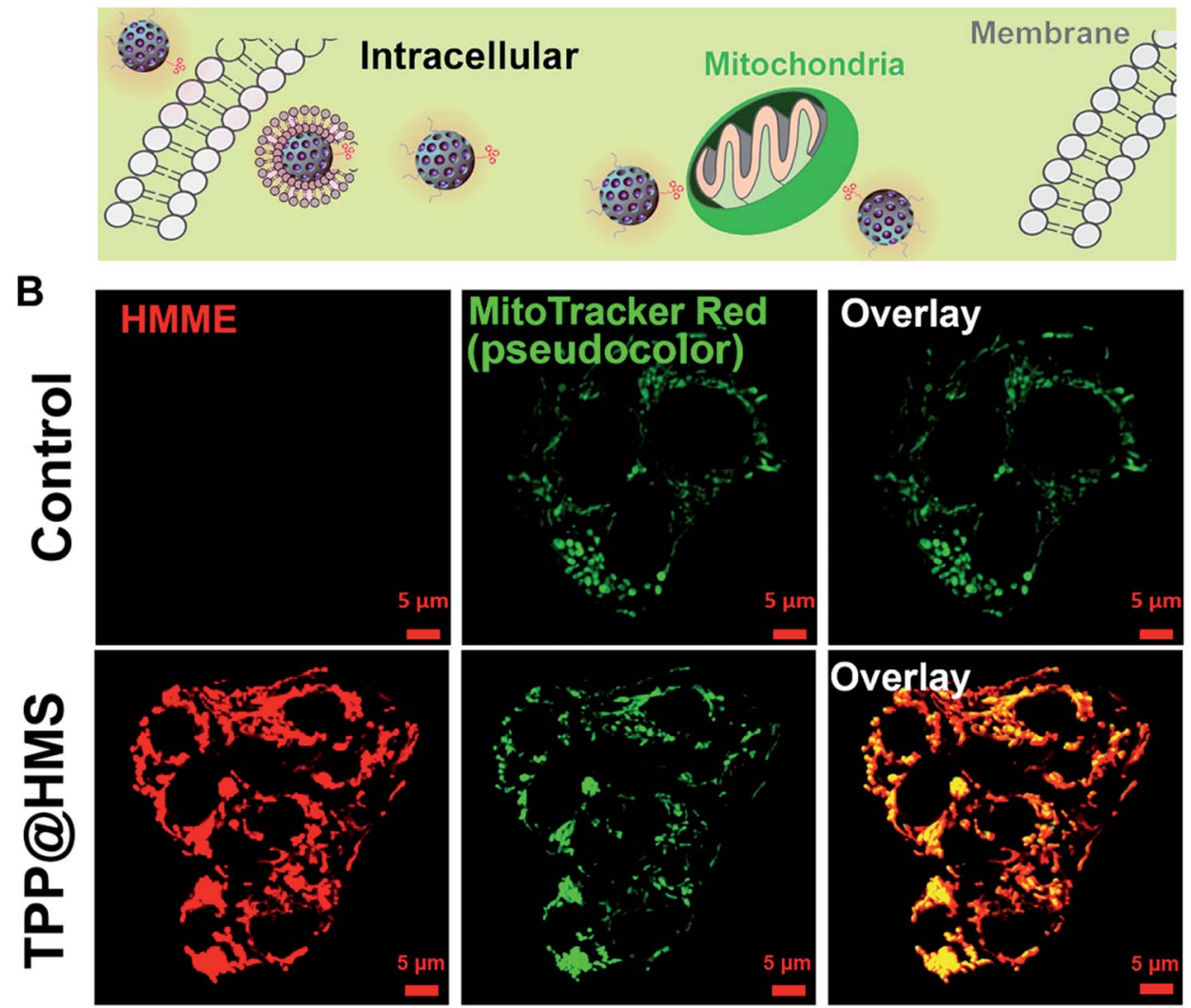

Fig. 2 (A) Schematic illustration of HepG2 cells with TPPaHMS. (B) Intracellular distribution of TPPaHMS in HepG2 cells after co-incubation for $6 \mathrm{~h}$, and imaged by CLSM. The intracellular mitochondria were stained by MitoTracker Red (green, pseudocolor). 
morphology of the cancer cells was also examined by CLSM after TPP@HMS treatment upon US $\left(1 \mathrm{WHz}, 0.75 \mathrm{~W} \mathrm{~cm}^{-2}\right)$ for 3 min. As shown in Fig. 3D, obvious mitochondrial swelling/ disintegration was detected in the TPP@HMS-treated HepG2 cells after US treatment compared to the control. Furthermore, the intracellular ROS were investigated using the green fluorescence indicator 2,7-dichlorodihydrofluorescein diacetate (DCFH-DA) (Fig. 3E). ${ }^{20-22}$ Green fluorescence with a much higher fluorescence intensity was obviously detected in TPP@HMS-treated HepG2 cells after US treatment compared to the PBS-treated cells. These results suggested that our prepared TPP@HMS could act as an active mitochondriaspecific SDT nanoagent for further treatment of cancer cells.

In the TME, hypoxia and acidic $\mathrm{pH}$ play an important role in the heterogeneity of tumors. ${ }^{24}$ The limited oxygen in the TME can diminish the SDT effect, which may cause tumor relapse after SDT treatment. In this study, we combined a Fenton-like agent as an oxygen-independent CDT approach to convert the $\mathrm{H}_{2} \mathrm{O}_{2}$ in the TME into $\cdot \mathrm{OH}$ to induce tumor cells apoptosis/necrosis and SDT, overcoming the above limitation for enhanced SDT efficiency. $\mathrm{Cu}^{2+}$ ions as a Fenton-like agent have been widely used in CDT therapy. ${ }^{19,20,25}$ To enhance the $\mathrm{Cu}^{2+}$ ion accumulation in tumors, the oral administration of $\mathrm{Cu}^{2+}$-containing compounds is a currently available approach that is commonly utilized in copper deficiency in clinic. Unfortunately, the systemic administration of $\mathrm{Cu}^{2+}$ compounds may induce heavy-metal poisoning. ${ }^{26,27}$ Herein, $\mathrm{Cu}^{2+}$ was coordinated with TPP@HMS via the $\mathrm{NH}_{2}$-medium coordination effect to form TPP-Cu@HMS at neutral pH according to our previously reported work. ${ }^{28}$ STEM-HAADF was performed to confirm the existence of $\mathrm{Cu}$ in TPP$\mathrm{Cu}$ @HMS. Fig. 4A shows the presence of $\mathrm{Cu}, \mathrm{N}, \mathrm{Si}, \mathrm{O}$, and $\mathrm{C}$ elements in TPP-Cu@HMS, and $\mathrm{Cu}$ was homogenously distributed throughout TPP-Cu@HMS, indicating the existence of $\mathrm{Cu}^{2+}$. Next, the bio-stability of TPP-Cu@HMS in physiological conditions (at $\mathrm{pH}$ 7.4) was investigated. The results showed $13.5 \%$ of $\mathrm{Cu}^{2+}$ was released from TPPCu@HMS after $24 \mathrm{~h}$ incubation, indicating its relative
A

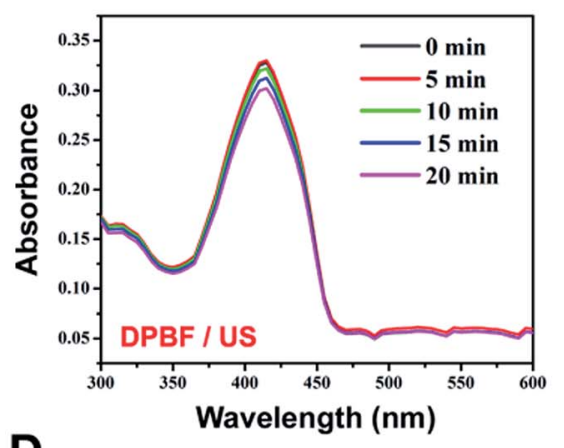

D

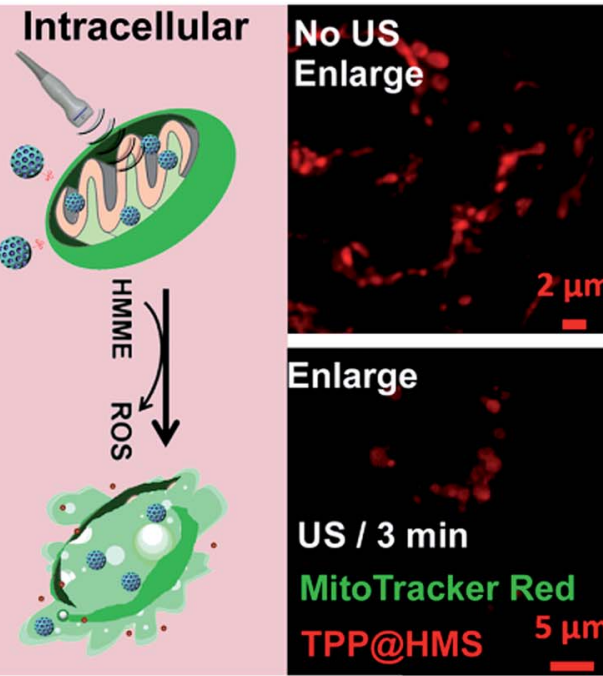

B

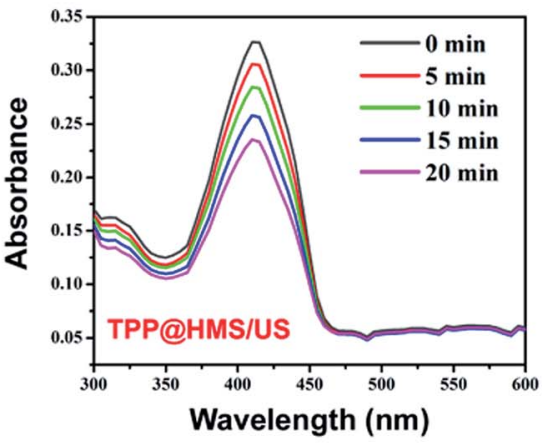

C

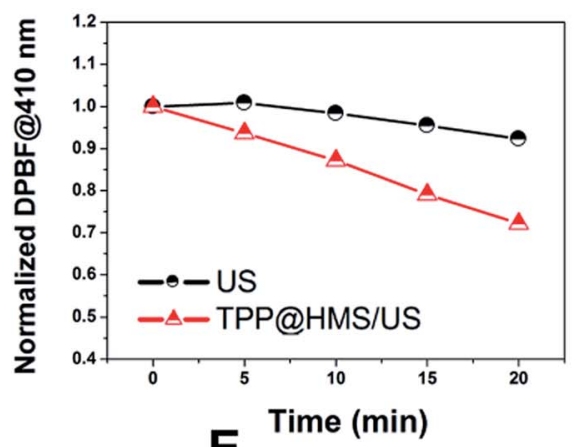

E
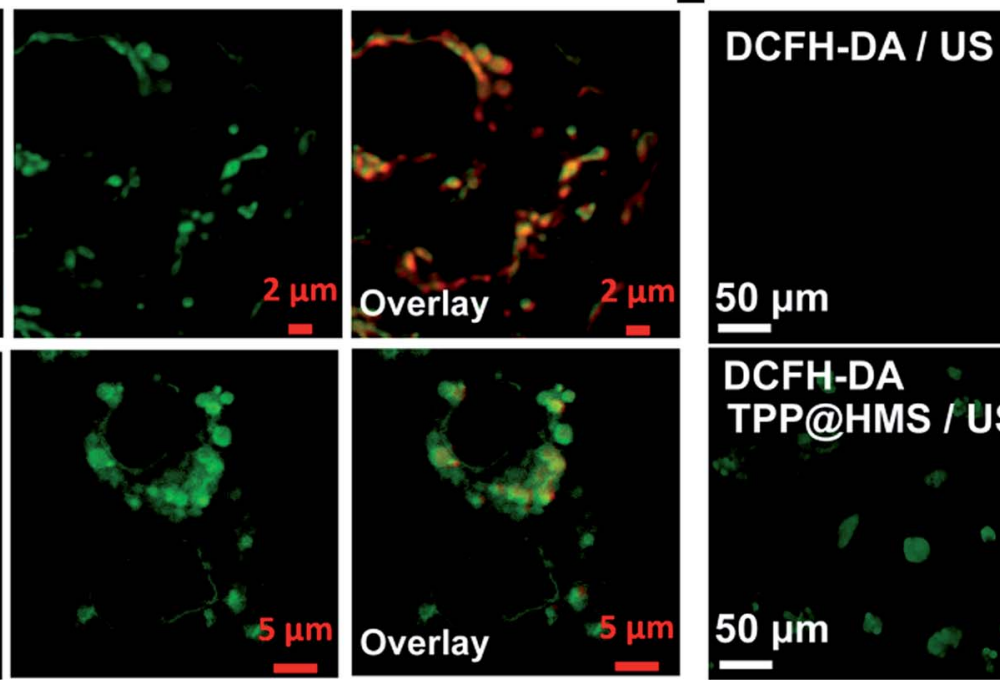
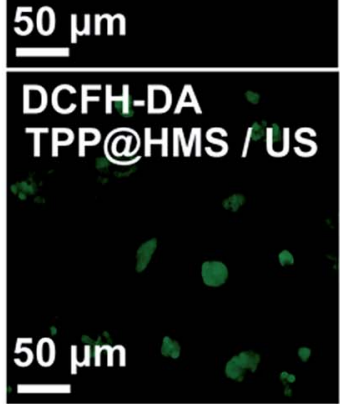

Fig. 3 Absorbance spectra of (A) DPBF with US $(100 \mu M)$ and (B) DPBF $(100 \mu M)$ in TPP@HMS with ROS generation upon US (1 MHz, 0.75 W $\mathrm{cm}^{-2}$ ) for different intervals, where DPBF with US is the positive control. (C) Normalized absorption coefficient of DPBF at $410 \mathrm{~nm}$ based on (A and B). (D) CLSM images of mitochondria morphology after HepG2 cells were treated with TPP(aHMS for $6 \mathrm{~h}$ with or without US $\left(0.75 \mathrm{~W} \mathrm{~cm}^{-2}, 3 \mathrm{~min}\right)$ treatment. (E) CLSM image of intracellular ROS generation by DCFH-DA detector after PBS or TPPaHMS-treated cells were exposed to US $\left(0.75 \mathrm{~W} \mathrm{~cm}^{-2}, 3 \mathrm{~min}\right)$ treatment. 
A

stability in physiological conditions (Fig. S1†). Afterwards, we further checked the catalytic performance of TPP-Cu@HMS in TMB $(250 \mu \mathrm{M})$ aqueous solution in the presence of $\mathrm{H}_{2} \mathrm{O}_{2}$. After co-incubation, the absorbance of TMB obviously increased in TPP-Cu@HMS with the presence of $\mathrm{H}_{2} \mathrm{O}_{2}(0.625 \mathrm{mM})$, and the color of solution changed to wathet blue due to the $\mathrm{Cu}^{2+}$ ions from TPP-Cu@HMS converting $\mathrm{H}_{2} \mathrm{O}_{2}$ to $\cdot \mathrm{OH}$. In contrast, the TMB solution in the presence of $\mathrm{H}_{2} \mathrm{O}_{2}$ without TPP-Cu@HMS did not show any change. Moreover, we checked the ROS generation of TPP-Cu@HMS in cancer cells using the DCFHDA indicator after co-incubation for $24 \mathrm{~h}$, and the results showed relatively high ROS generation in TPP-Cu@HMStreated HepG2 cells compared with the control groups. These results are similar to the intracellular $\cdot \mathrm{OH}$ generation from TPP-Cu@HMS without US (Fig. S2 $\dagger$ ). Thus, the results indicate that TPP-Cu@HMS can act as an activatable SDT-CDT nanoagent for further cancer therapy.

The mitochondrial membrane permeability (MMP) is essential in mitochondria apoptosis and cell death. Encouraged by the above results, we investigated the mitochondrial membrane $\left(\Delta \psi_{\mathrm{m}}\right)$ using the JC-1 dye detection kit (the potential sensitive indicator) and imaging by CLSM. As illustrated in Fig. 5, the JC-1 monomers with green fluorescence were more obviously and increased in the TPPCu@HMS-treated HepG2 cells after 1 WHz US $(0.75 \mathrm{~W}$ $\mathrm{cm}^{-2}$ ) for $3 \mathrm{~min}$. The ratio of JC-1 aggregates with red fluorescence/JC-1 monomers with green fluorescence was reduced, indicating the high in situ $\operatorname{ROS}\left({ }^{1} \mathrm{O}_{2}\right.$ and $\left.\cdot \mathrm{OH}\right)$ generation and accurate photodamage on mitochondria.
B

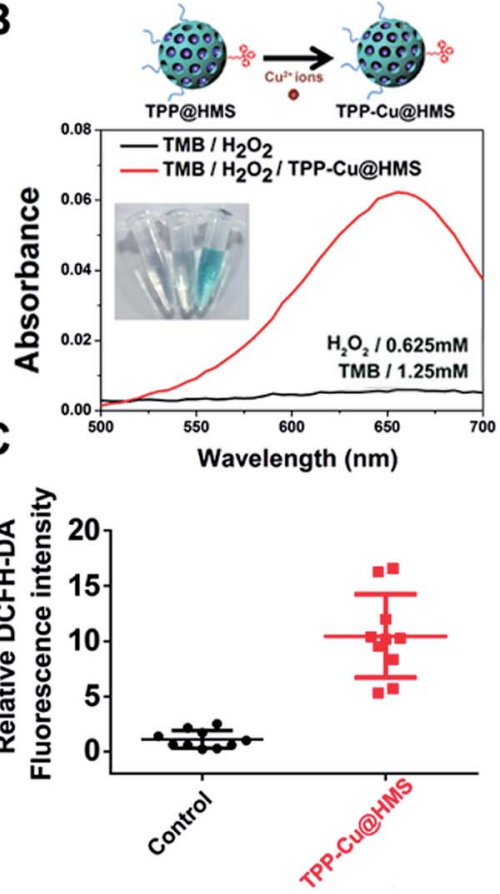

However, the JC-1 monomer fluorescence intensity was low in the TPP-Cu@HMS-treated HepG2 cells alone. In contrast, the HepG2 cells without any treatment showed overwhelming red fluorescence. Noteworthily, green fluorescence was observed in the US-treated HepG2 cells in the absence of TPP-Cu@HMS mainly due to the cavitation effect of ultrasound. ${ }^{22,23}$ Next, the synergistic therapeutic efficiency of TPPCu@HMS was investigated by PI dead staining and analyzed through fluorescence microscopy. A significant amount of dead cells with red fluorescence was obviously detected in the TPP-Cu@HMS-treated HepG2 cells after received $1 \mathrm{WHz}$ US $\left(0.75 \mathrm{~W} \mathrm{~cm}^{-2}\right)$ for $3 \mathrm{~min}$ compared to the TPP-Cu@HMStreated cells alone and US alone (Fig. 6A). Meanwhile, to quantify the TPP-Cu@HMS cytotoxicity after US treatment, Cell Counting Kit-8 assay (CCK-8) was performed. As illustrated in Fig. 6B, the cell viability of the TPP@HMS-treated cancer cells alone showed no obvious cytotoxicity after $24 \mathrm{~h}$ incubation, and the mortality rate was very low even with the concentration of TPP@HMS up to $0.3 \mathrm{mg} \mathrm{mL} \mathrm{m}^{-1}$, indicating that our prepared TPP@HMS has good biocompatibility. In contrast, the viable cells of TPP-Cu@HMS-treated HepG2 cells upon $1 \mathrm{WHz}$ US $\left(0.75 \mathrm{~W} \mathrm{~cm}^{-2}\right)$ for $3 \mathrm{~min}$ significantly decreased to $56.6 \%$, suggesting the synergistic treatment of TPP-Cu@HMS compared to TPP-Cu@HMS-treated HepG2 cells alone without US treatment and HMS-treated HepG2 cells with US treatment. Overall, the results highlight the potential of our prepared TPP-Cu@HMS as a powerful therapeutic agent for enhanced mitochondria-specific cancer therapy. 
A

B
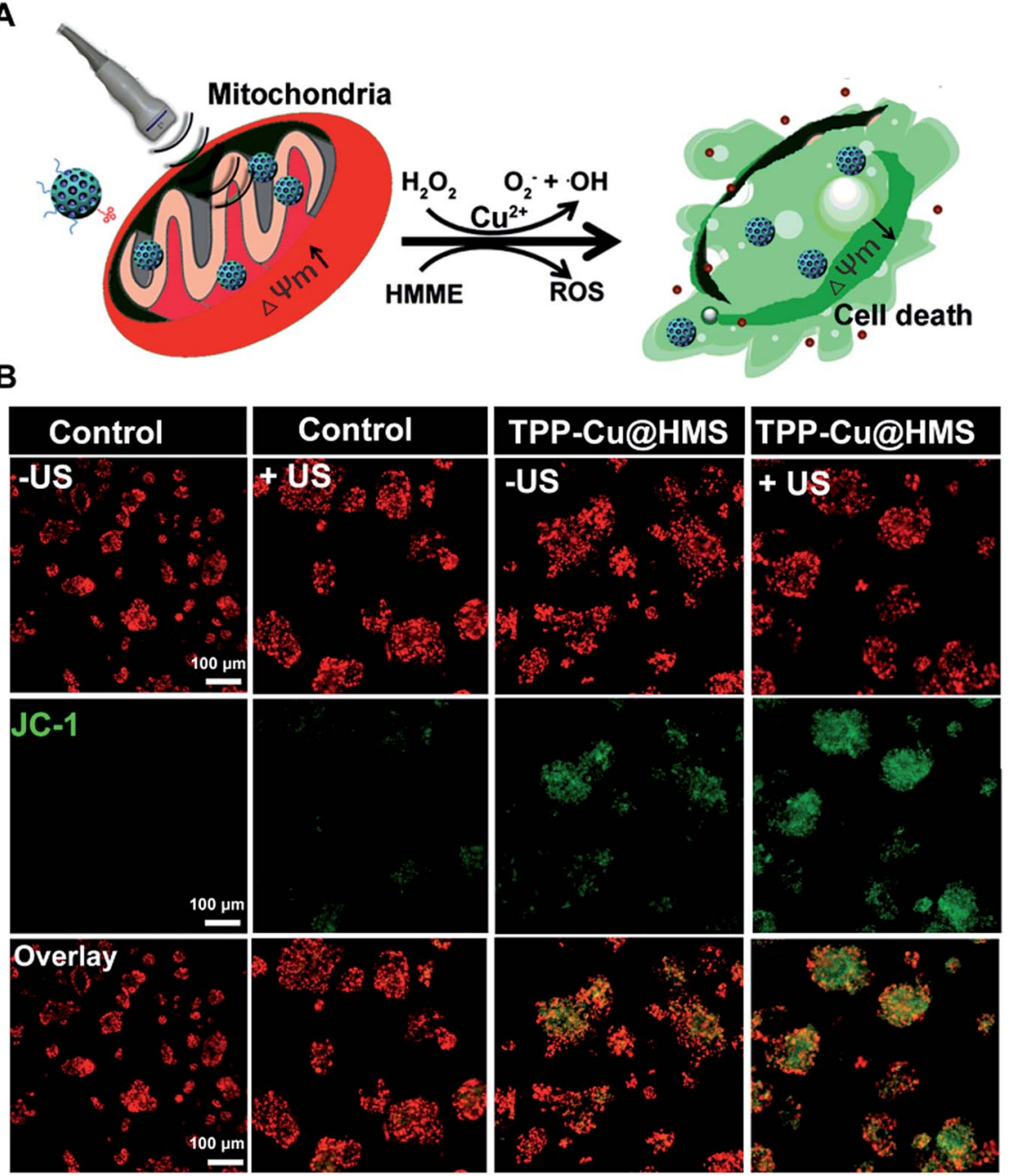

Fig. 5 (A) Schematic illustration of the mitochondrial membrane potential change $\left(\Delta \Psi_{m}\right)$ after US. (B) CLSM images of JC-1-stained (green channel), and TPP-CuaHMS (red channel)-treated HepG2 cells under US treatment.

A

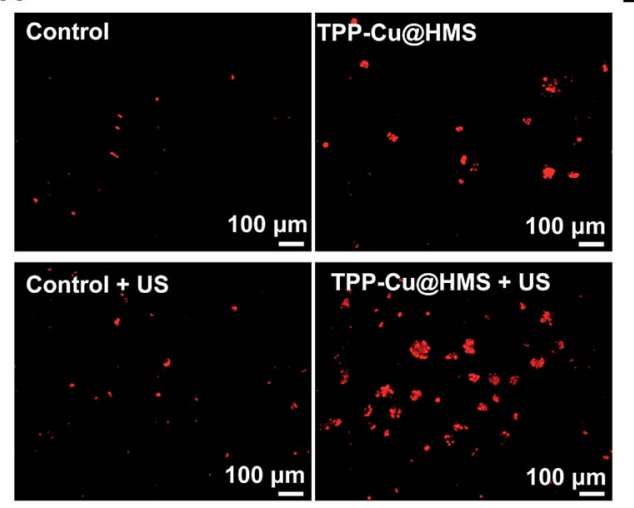

B

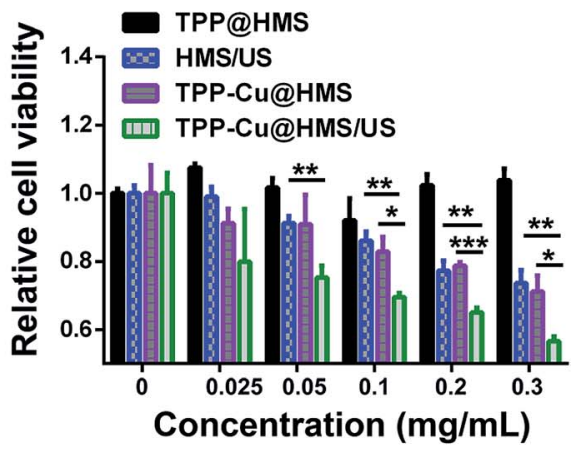

Fig. 6 (A) Localized photo-killing effects of TPP@HMS or TPP-Cu@HMS with or without US (1 MHz, $\left.0.75 \mathrm{~W} \mathrm{~cm}^{-2}\right)$ on HepG2 cells. (B) Cell viability of the HMS, TPPAHMS or TPP-CuQHMS treated HepG2 cells with or without US $\left(1 \mathrm{MHz}, 0.75 \mathrm{~W} \mathrm{~cm}^{-2}\right)$ for 3 min, where the cell viability was determined using the CCK-8 assay. The statistical analysis was performed with the two-tailed paired Student's $t$-test, where $* p<0.05, * * p 0.01$, $* * * p<0.001(n=4)$. 


\section{Conclusion}

In summary, we introduced a promising therapeutic agent and integration of SDT and CDT to in situ generate $\mathrm{ROS}\left({ }^{1} \mathrm{O}_{2}\right.$ and - $\mathrm{OH}$ ) for mitochondria-specific cancer therapy. TPP-Cu@HMS was easily obtained and endowed with mitochondria-specific targeting ability by decoration with TPP-COOH ligands. After US treatment, TPP-Cu@HMS generated a high amount of ${ }^{1} \mathrm{O}_{2}$ and $\cdot \mathrm{OH}$ in the mitochondria and effectively induced mitochondrial disintegration and damage. According to the in vitro therapeutic data, TPP-Cu@HMS effectively induced apoptosis in cancer cells compared to the individual treatments. Therefore, we highlight the current strategy as a promising approach for enhanced cancer therapy.

\section{Conflicts of interest}

The authors declare no competing financial interest.

\section{Acknowledgements}

Kecan Lin and Ziguo Lin contributed equally to this work. The manuscript was written through contributions of all authors. All authors have given approval to the final version of the manuscript. This work was supported by the Natural Science Foundation of China (Grant No. 61775037, 61805041). Bethune Aishikun Surgical Excellence Fund and Joint Funds for the Innovation of Science and Technology, Fujian province (Grant No. 2018Y9119).

\section{Notes and references}

1 M. L. Boland, A. H. Chourasia and K. F. Macleod, Front. Oncol., 2013, 3, 292.

2 M. Jeena, L. Palanikumar, E. M. Go, I. Kim, M. G. Kang, S. Lee, S. Park, H. Choi, C. Kim and S.-M. Jin, Nat. Commun., 2017, 8, 26.

3 D. A. Bader, S. M. Hartig, V. Putluri, C. Foley, M. P. Hamilton, E. A. Smith, P. K. Saha, A. Panigrahi, C. Walker and L. Zong, Nature Metabolism, 2019, 1, 70.

4 M. Ott, V. Gogvadze, S. Orrenius and B. Zhivotovsky, Apoptosis, 2007, 12, 913-922.

5 O. M. Koval, E. K. Nguyen, V. Santhana, T. P. Fidler, S. C. Sebag, T. P. Rasmussen, D. J. Mittauer, S. Strack, P. C. Goswami and E. D. Abel, Sci. Signaling, 2019, 12, 1439.

6 Y. Chen, W. Ai, X. Guo, Y. Li, Y. Ma, L. Chen, H. Zhang, T. Wang, X. Zhang and Z. Wang, Small, 2019, 1902352.

7 A. Vadivelmurugan, R. Anbazhagan, V. Arunagiri, J. Y. Lai and H. C. Tsai, RSC Adv., 2019, 9, 25592-25601.
8 Y. Zheng, X. Ji, B. Yu, K. Ji, D. Gallo, E. Csizmadia, M. Zhu, M. R. Choudhury, L. K. C. De La Cruz and V. Chittavong, Nat. Chem., 2018, 10, 787.

9 W. Zhang, X. Hu, Q. Shen and D. Xing, Nat. Commun., 2019, 10, 1704.

10 L. Zhang, H. Yi, J. Song, J. Huang, K. Yang, B. Tan, D. Wang, N. Yang, Z. Wang and X. Li, ACS Appl. Mater. Interfaces, 2019, 11, 9355-9366.

11 F. Zhou, S. Wu, B. Wu, W. R. Chen and D. Xing, Small, 2011, 7, 2727-2735.

12 P. Yuan, X. Mao, X. Wu, S. S. Liew, L. Li and S. Q. Yao, Angew. Chem., 2019, 131, 7739-7743.

13 X.-Q. Wang, M. Peng, C.-X. Li, Y. Zhang, M. Zhang, Y. Tang, M.-D. Liu, B.-R. Xie and X.-Z. Zhang, Nano Lett., 2018, 18, 6804-6811.

14 X. Lin, J. Song, X. Chen and H. Yang, Angew. Chem., 2019, DOI: 10.1002/ange.201906823.

15 S. G. Rhee, Science, 2006, 312, 1882-1883.

16 X. Lin, Y. Qiu, L. Song, S. Chen, X. Chen, G. Huang, J. Song, X. Chen and H. H. Yang, Nanoscale Horiz., 2019, 4, 747-756.

17 K. L. Zhang, Y. J. Wang, J. Sun, J. Zhou, C. Xing, G. Huang, J. Li and H. H. Yang, Chem. Sci., 2019, 10, 1555-1561.

18 D. Zhang, Z. Cai, N. Liao, S. Lan, M. Wu, H. Sun, Z. Wei, J. Li and X. Liu, Chem. Sci., 2018, 9, 7390-7399.

19 D. Zhang, Z. Lin, S. Lan, H. Sun, Y. Zeng and X. Liu, Mater. Chem. Front., 2019, 3, 656-663.

20 L. S. Lin, J. Song, L. Song, K. Ke, Y. Liu, Z. Zhou, Z. Shen, J. Li, Z. Yang, W. Tang, G. Niu, H. H. Yang and X. Chen, Angew. Chem., 2018, 57, 4902-4906.

21 D. Zhang, Y. Zheng, Z. Lin, S. Lan, X. Zhang, A. Zheng, J. Li, G. Liu, H. H. Yang, X. Liu and J. Liu, Small, 2019, 1902636.

22 T. C. Lei, G. F. Glazner, M. Duffy, L. Scherrer, S. Pendyala, B. Li, X. Wang, H. Wang and Z. Huang, Photodiagn. Photodyn. Ther., 2012, 9, 232-242.

23 J. Shi, R. Ma, L. Wang, J. Zhang, R. Liu, L. Li, Y. Liu, L. Hou, X. Yu and J. Gao, Int. J. Nanomed., 2013, 8, 2361.

24 S. Lan, Z. Lin, D. Zhang, Y. Zeng and X. Liu, ACS Appl. Mater. Interfaces, 2019, 11, 9804-9813.

25 L. Lin, T. Huang, J. Song, X. Y. Ou, Z. Wang, H. Deng, R. Tian, Y. Liu, J. F. Wang and Y. Liu, J. Am. Chem. Soc., 2019, 141, 9937-9945.

26 W. Wu, L. Yu, Q. Jiang, M. Huo, H. Lin, L. Wang, Y. Chen and J. Shi, J. Am. Chem. Soc., 2019, 141, 11531-11539.

27 Z. Chen, H. Meng, G. Xing, C. Chen, Y. Zhao, G. Jia, T. Wang, H. Yuan, C. Ye and F. Zhao, Toxicol. Lett., 2006, 163, 109-120. 28 D. Zhang, A. Zheng, J. Li, M. Wu, L. Wu, Z. Wei, N. Liao, X. Zhang, Z. Cai, H. H. Yang, G. Liu, X. Liu and J. Liu, Theranostics, 2017, 7, 164-179. 